\title{
Hydroxycarboxylic acid receptor 2 mediates dimethyl fumarate's protective effect in EAE
}

\author{
Hui Chen, ${ }^{1}$ Julian C. Assmann, ${ }^{1}$ Antje Krenz, ${ }^{2}$ Mahbubur Rahman, ${ }^{1}$ \\ Myriam Grimm, ${ }^{3}$ Christian M. Karsten, ${ }^{4}$ Jörg Köhl, ${ }^{4}$ Stefan Offermanns, ${ }^{3,5}$ \\ Nina Wettschureck, ${ }^{3,5}$ and Markus Schwaninger ${ }^{1}$ \\ ${ }^{1}$ Institute of Experimental and Clinical Pharmacology and Toxicology, University of Lübeck, Lübeck, Germany. \\ IInstitute of Pharmacology, University of Heidelberg, Heidelberg, Germany. \\ ${ }^{3}$ Department of Pharmacology, Max Planck Institute for Heart and Lung Research, Bad Nauheim, Germany. \\ ${ }^{4}$ Institute of Systemic Inflammation Research, University of Lübeck, Lübeck, Germany. ${ }^{5}$ Medical Faculty, Goethe University, Frankfurt, Germany.
}

\begin{abstract}
Taken orally, the drug dimethyl fumarate (DMF) has been shown to improve functional outcomes for patients with MS; however, it is unclear how DMF mediates a protective effect. DMF and, more so, its active metabolite, monomethyl fumarate, are known agonists of the hydroxycarboxylic acid receptor $2\left(\mathrm{HCA}_{2}\right)$, a G proteincoupled membrane receptor. Here, we evaluated the contribution of $\mathrm{HCA}_{2}$ in mediating the protective effect afforded by DMF in EAE, a mouse model of MS. DMF treatment reduced neurological deficit, immune cell infiltration, and demyelination of the spinal cords in wild-type mice, but not in $\mathrm{Hca2}^{-/-}$mice, indicating that $\mathrm{HCA}_{2}$ is required for the therapeutic effect of DMF. In particular, DMF decreased the number of infiltrating neutrophils in a $\mathrm{HCA}_{2}$-dependent manner, likely by interfering with neutrophil adhesion to endothelial cells and chemotaxis. Together, our data indicate that $\mathrm{HCA}_{2}$ mediates the therapeutic effects of DMF in EAE. Furthermore, identification of $\mathrm{HCA}_{2}$ as a molecular target may help to optimize MS therapy.
\end{abstract}

\section{Introduction}

Recently, orally available drugs that modify the disease process of MS have come into routine use in clinical practice. Dimethyl fumarate (DMF) stands out as a compound that is more effective than standard parenteral treatment $(1,2)$. DMF has been used to treat psoriasis since the 1990s; during that time, the drug has had a well-documented safety record (3). Of its adverse effects, flushing and gastrointestinal side effects at the beginning of therapy are most common.

Although the clinical efficacy of DMF has been established and the drug has been approved by the FDA and European Medicines Agency, its mode of action is still incompletely understood. In EAE, an animal model of MS (4), DMF has shown immunomodulatory and neuroprotective effects and improved the clinical score (5-7). DMF is rapidly metabolized to monomethyl fumarate (MMF). DMF and MMF covalently modify glutathione and KEAP-1, the inhibitor of the antioxidant transcription factor nuclear factor erythroid-derived 2-related factor 2 (NRF2), and induce hemoxygenase-1 (HMOX1), NADP(H) quinoline oxidoreductase-1 (NQO1), and other genes $(6,7)$. Whether the covalent modifications occur in vivo and eventually ameliorate MS pathology is still unclear.

Recently, the carboxylic acid MMF was shown to be a potent agonist of the hydroxycarboxylic acid receptor $2\left(\mathrm{HCA}_{2}\right.$; GPR109A) (8). This $\mathrm{G}_{\mathrm{i}}$-coupled membrane receptor mediates the lipid-lowering effects of nicotinic acid but is also expressed by keratinocytes and immune cells and especially by neutrophils (9). In this study, we aimed to elucidate the role of $\mathrm{HCA}_{2}$ in the disease-modifying action of DMF in EAE. Our data suggest that $\mathrm{HCA}_{2}$ is an important drug target for the treatment of MS.

Authorship note: Nina Wettschureck and Markus Schwaninger contributed equally to this work.

Conflict of interest: The authors have declared that no conflict of interest exists. Citation for this article: J Clin Invest. 2014;124(5):2188-2192. doi:10.1172/JCI72151.

\section{Results and Discussion}

To investigate the potential role of $\mathrm{HCA}_{2}$ in the protective effect afforded by DMF, we used wild-type and $\mathrm{Hca}^{2--}$ mice that show no overt phenotype if unchallenged (10). Starting 3 days postimmunization (dpi), we administered vehicle or DMF $(30 \mathrm{mg} / \mathrm{kg}$, twice per day) by gavage. DMF treatment improved the motor score of wild-type mice but lost its effect in $\mathrm{Hca}^{-/-}$mice (Figure 1A). DMF also reduced the area under the curve of the motor deficit, the peak scores, and the day of disease onset in wild-type mice but not in $\mathrm{Hca}^{-/-}$mice (Figure 1, B-D). We reproduced the experiment twice with mice coming from 2 different animal facilities and with littermates in one case (Supplemental Figure 1, A and B; supplemental material available online with this article; doi:10.1172/ JCI72151DS1). Although the efficacy of DMF varied among experimental series, it always depended on $\mathrm{HCA}_{2}$.

On dpi 28, we evaluated the histopathology of the spinal cord (for the clinical scores of these mice, see Supplemental Figure 1A). Treating wild-type mice with DMF lowered the number of inflammatory cells (Figure 1, E and F). In contrast, there was no significant difference in the number of inflammatory cells between vehicle- and DMF-treated $\mathrm{Hca}^{-/-}$mice (Figure $1, \mathrm{E}$ and F). Furthermore, the area of demyelination was reduced by DMF treatment in wild-type mice but not $\mathrm{Hca}^{-/-}$mice (Figure 1, G and H). These data show that $\mathrm{HCA}_{2}$ is required for the protective effect of DMF in EAE.

As $\mathrm{HCA}_{2}$ is expressed in immune cells, such as macrophages and neutrophils (11), we characterized the action of DMF on immune cell infiltration into the spinal cord by using flow cytometry on dpi 17 (Figure 2). DMF treatment reduced the number of $\mathrm{CD} 45^{+} \mathrm{Ly}-6 \mathrm{G}^{+}$neutrophils in the spinal cords of wild-type mice but had no effect in $\mathrm{Hca}^{--/}$mice (Figure 2D). In addition, a nonsignificant trend toward lower numbers of $\mathrm{CD} 4^{+} \mathrm{T}$ cells, $\mathrm{CD} 11 \mathrm{~b}^{+}$macrophages, and $\mathrm{CD} 11 \mathrm{c}^{+}$dendritic cells was observed in wild-type mice but not in $\mathrm{Hca2}^{-/-}$mice (Figure 2, A, C, and E). 
A

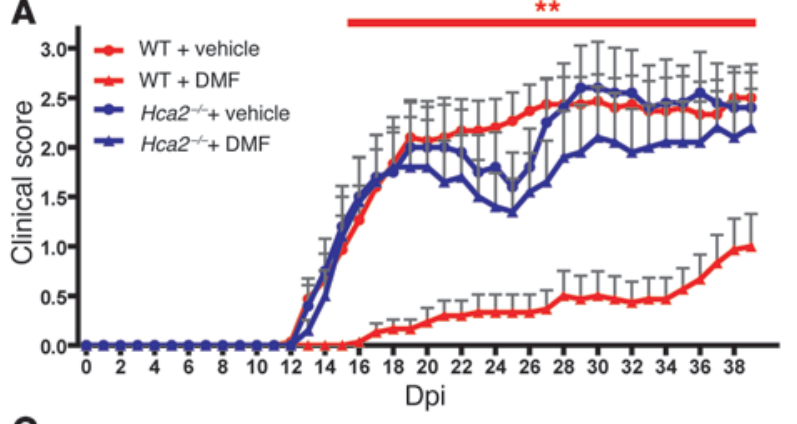

C

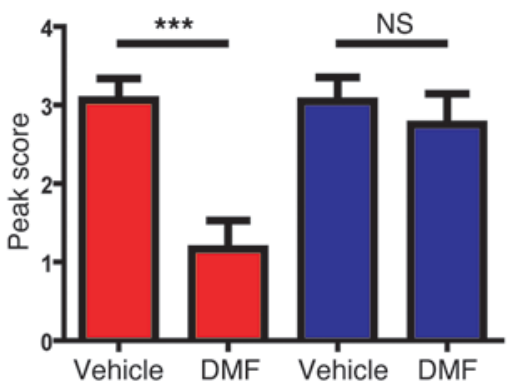

E

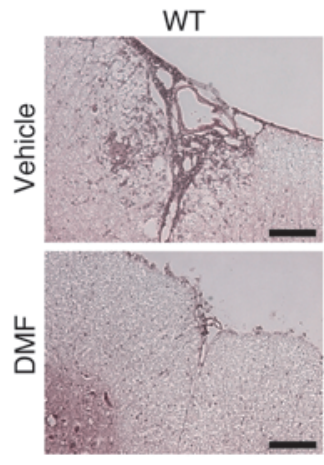

G

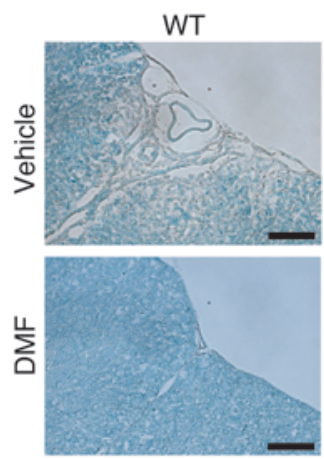

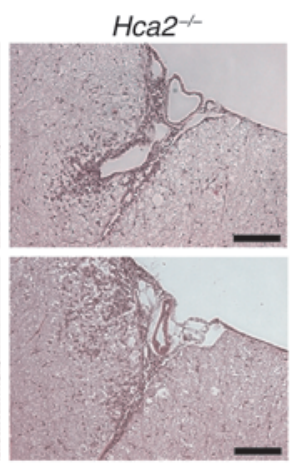

$\mathrm{Hca}^{-/-}$
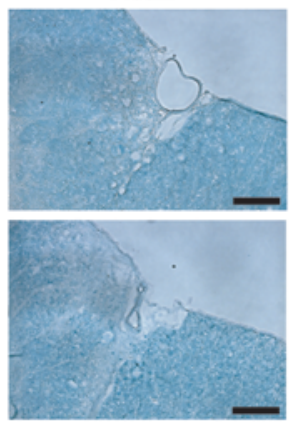

B
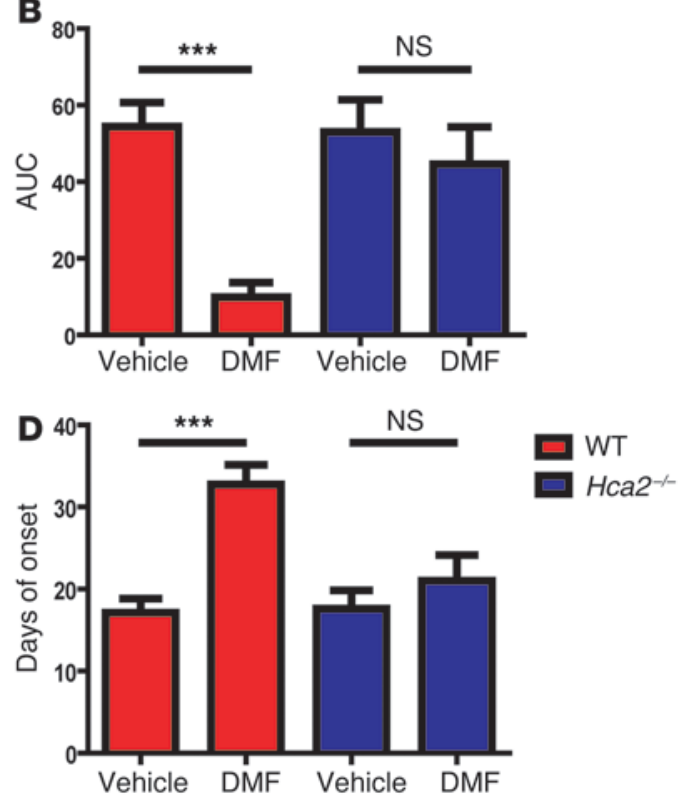

F

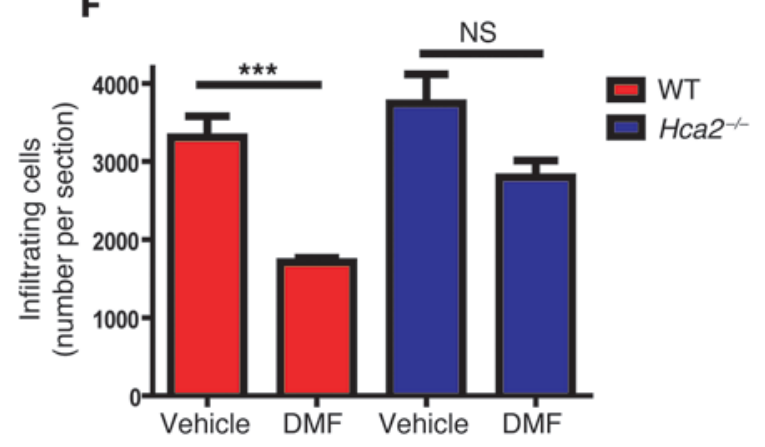

H

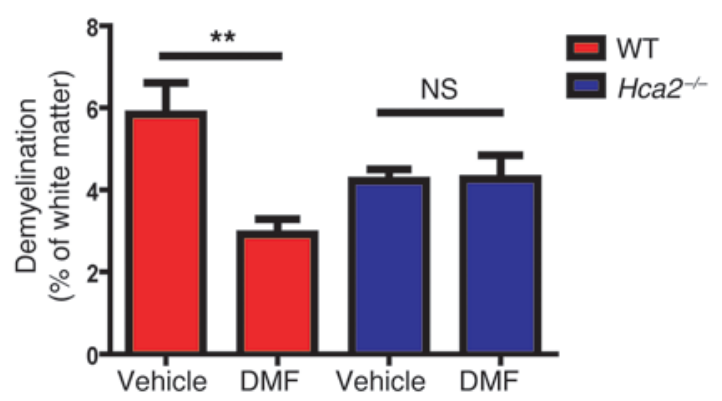

Figure 1

DMF treatment attenuates the severity of EAE in wild-type mice but not in $\mathrm{Hca}^{-/-}$mice. (A) EAE clinical scores for wild-type and $\mathrm{Hca2}{ }^{-/-}$mice ( $n=10-15$ per group) that were treated orally with vehicle or DMF (30 mg/kg body weight, twice per day). The transient partial remission observed in this experiment in the $\mathrm{Hca}^{-/-}$groups was not reproduced in other experiments and therefore is not a general feature of $\mathrm{Hca}^{-/-}$mice (see Supplemental Figure 1, A and B). ${ }^{* *} P<0.01$ for comparison between "WT + Vehicle" and "WT + DMF" by nonparametric Mann-Whitney test. (B-D) DMF reduced the area under the curve (AUC), the peak score, and the day of disease onset in wild-type mice but not in Hca2 ${ }^{-/-}$mice. $P=0.0001$, Kruskal-Wallis for AUC; $P=0.0003$, Kruskal-Wallis test for peak scores; $P=0.0002$, Kruskal-Wallis test for day of onset. ${ }^{* *} P<0.001$ (Dunn's multiple comparison test). NS, nonsignificant. (E and $\mathbf{F}$ ) Immune cell infiltration was assessed by H\&E staining of spinal cord sections on dpi $28(n=6-7) . P=0.0002$ (2-way ANOVA for drug treatment); ${ }^{* * *} P<0.001$ (Bonferroni post-hoc test). Scale bar: $100 \mu \mathrm{m}$. (G and $\mathbf{H}$ ) White matter demyelination was determined by Luxol Fast Blue staining of spinal cord sections on dpi 28 $(n=6-7) . P<0.0125$ (2-way ANOVA for drug treatment); ${ }^{*} P<0.01$ (Bonferroni post-hoc test). Scale bar: $100 \mu \mathrm{m}$. (E-H) Clinical scores of mice are given in Supplemental Figure $1 \mathrm{~A}$. Data represent mean \pm SEM. 
A

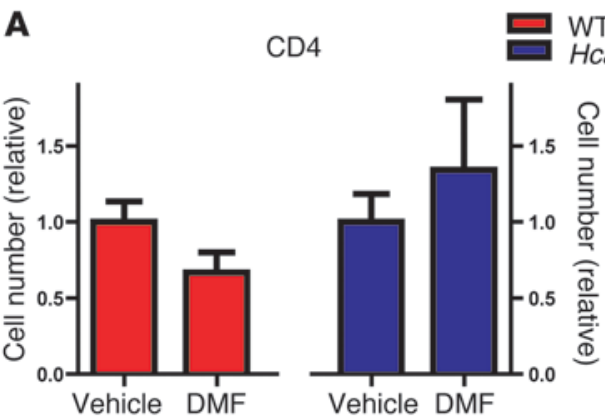

C

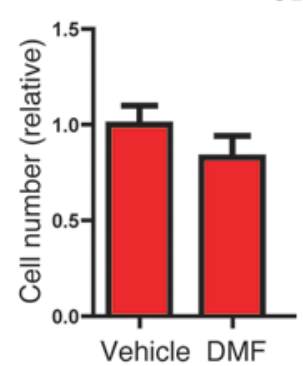

E

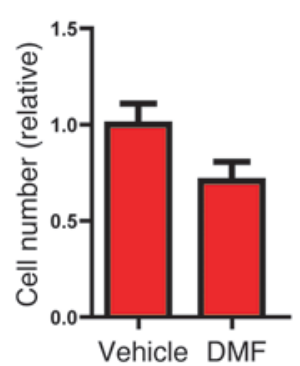

conit

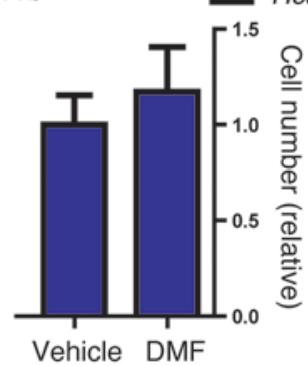

Vehicle DMF
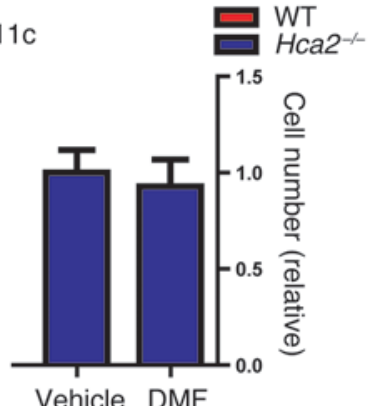

B

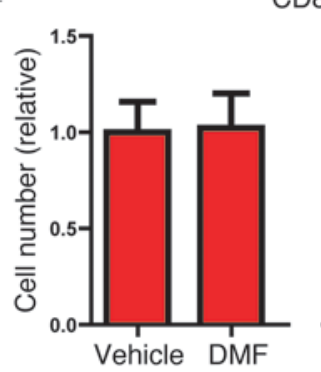

CD8

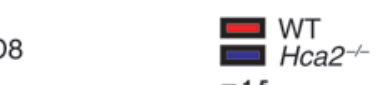

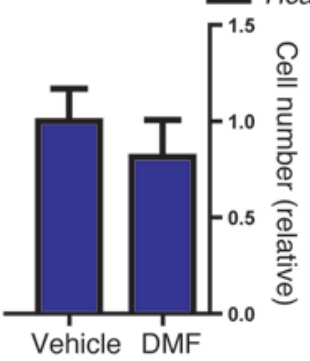

D

Ly-6G
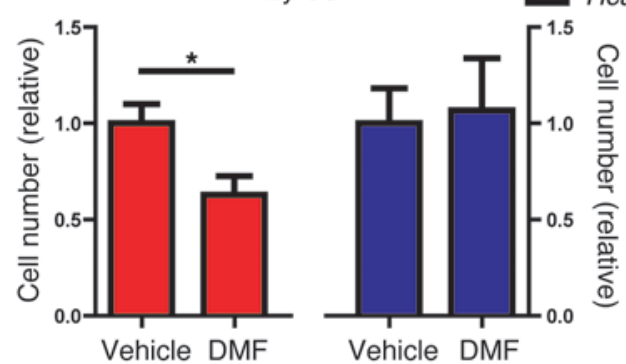

Figure 2

DMF treatment reduces neutrophil infiltration into the spinal cords in wild-type mice but not in $\mathrm{Hca2}^{-1-}$ mice with EAE. The number of (A) $\mathrm{CD}_{45}{ }^{+} \mathrm{CD} 4{ }^{+} \mathrm{T}$ helper cells, (B) $\mathrm{CD} 45^{+} \mathrm{CD} 8^{+} \mathrm{T}$ cytotoxic cells, (C) $\mathrm{CD} 45^{+} \mathrm{CD} 11 \mathrm{~b}^{+}$macrophages, (D) CD45+Ly-6G+ neutrophils, and $(\mathrm{E}) \mathrm{CD}_{4} 5^{+} \mathrm{CD} 11 \mathrm{c}^{+}$dendritic cells in the spinal cord was quantified by flow cytometry on dpi 17. DMF or vehicle were given orally (30 mg/kg body weight, twice per day) starting from dpi 3. Results are expressed relative to the vehicle group of the same genotype. Data are mean \pm SEM from 14 to 15 mice per group. ${ }^{*} P<0.05$ (Student's $t$ test with Bonferroni correction).

In EAE, neutrophils infiltrate the CNS and have been shown recently to play an important role in the disease, suggesting that reduced neutrophil infiltration may underlie the protective effect of DMF (12-15). To confirm that the Hca2 locus is expressed in mouse neutrophils, we made use of the reporter mouse line Hca2 ${ }^{\text {mRFP }}$, in which expression of the monomeric red fluorescent protein (mRFP) is driven by the Hca2 locus (16). Peritoneal neutrophils from this mouse line were mRFP-positive, indicating that they express $\mathrm{HCA}_{2}$ (Figure 3, A-C). In addition, neutrophils that infiltrated spinal cords in EAE were mRFP-positive. mRFP-positive cells that were not stained by anti-polymorphonuclear leukocyte (anti-PMN) most likely represent macrophages or microglia (Figure 3, D-F). $\mathrm{HCA}_{2}$ is coupled to $\mathrm{G}_{\mathrm{i}}$-type $\mathrm{G}$ proteins (10). As other $\mathrm{G}_{\mathrm{i}}$-coupled receptors are able to interfere with neutrophil adhesion to endothelial cells and with neutrophil migration (17), we hypothesized that DMF treatment may reduce adhesion and chemotaxis of neutrophils by activating $\mathrm{HCA}_{2}$. Because DMF is immediately hydrolyzed to MMF in the body (18), we used MMF in vitro. When purified neutrophils were added to cultured brain endothelial bEnd. 3 cells, pretreatment of endothelial cells with TNF increased the number of adherent neutrophils (Figure 3G). Interestingly, MMF reduced the number of wild-type neutrophils adhering to TNF-stimulated endothelial cells in a concentration-dependent manner (Supplemental Figure 2) but had no effect on $\mathrm{Hca}^{-/-}$neutrophil adhesion (Figure 3G). These experiments demonstrated that MMF is able to inhibit neutrophil adhesion to brain endothelial cells by activating $\mathrm{HCA}_{2}$. As the next step in neutrophil recruitment, we investigated chemotaxis. In a transmigration assay, MMF partially blocked the migration of wild-type neutrophils toward the chemokine CXCL2. However, in neutrophils from $\mathrm{Hca} 2^{-/-}$mice, MMF had no effect on the chemotactic activity of CXCL2. A recent report showed that, in human neutrophils, nicotinic acid induces cell death, and this effect was linked to the $\mathrm{HCA}_{2}$ receptor (19). MMF also slightly induced cell death in wild-type neutrophils in vitro but had no effect in $\mathrm{Hca2^{-/- }}$ neutrophils (Supplemental Figure 3A). DMF treatment did not affect neutrophil counts in the blood of mice (Supplemental Figure 3B), suggesting that, if DMF treatment triggered apoptosis in circulating neutrophils, the effect was balanced by the mobilization or distribution of neutrophils. Thus, it is unclear whether the small $\mathrm{HCA}_{2}$-dependent induction of cell death by MMF contributes to the beneficial effect in EAE.

DMF has been shown to activate the antioxidant transcription factor NRF2 and to induce its target genes, NQO1 and HMOX1 $(6,7)$. To investigate whether $\mathrm{HCA}_{2}$ is required for this effect, we treated peritoneal macrophages and primary microglia that express $\mathrm{HCA}_{2}$ (data not shown) with MMF in vitro. In accordance with previous reports, MMF induced Nqo1 and Hmox1 in wild- 

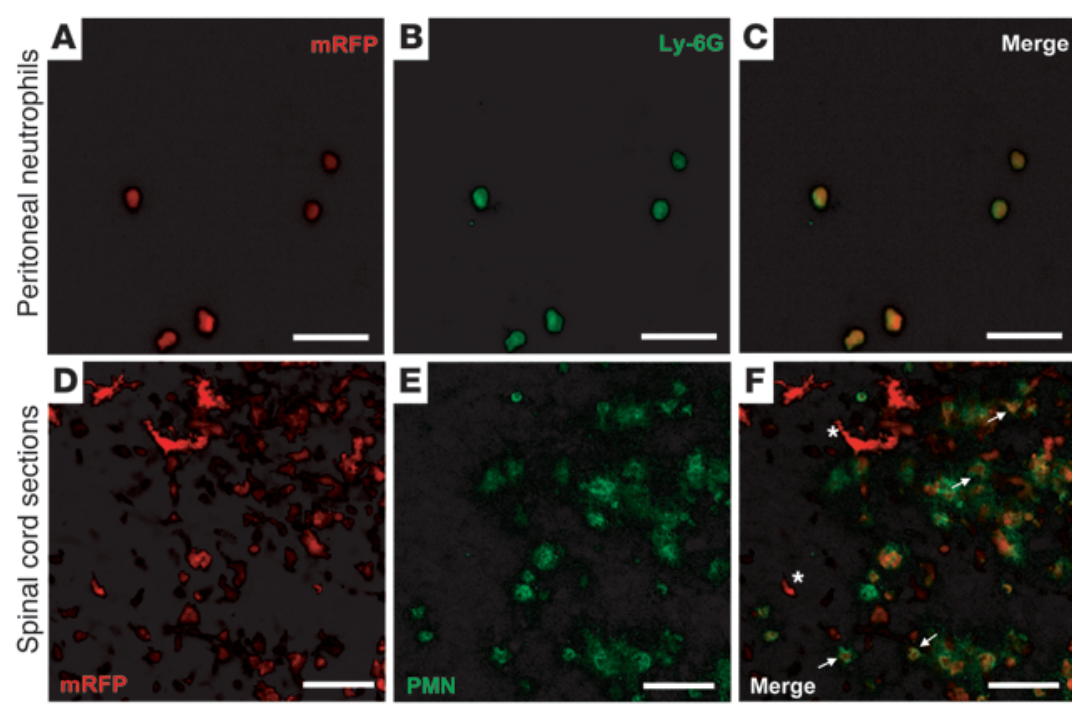

G

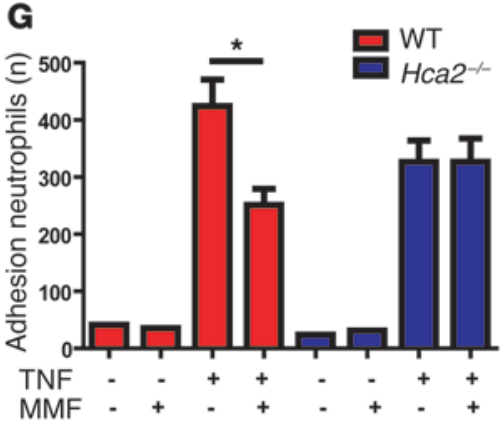

H

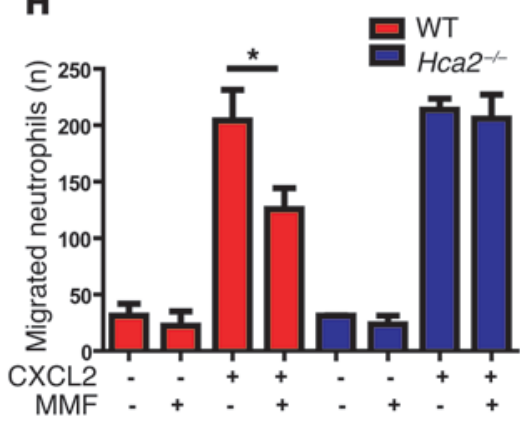

Figure 3

MMF inhibits neutrophil adhesion and migration via $\mathrm{HCA}_{2}$. (A-F) Neutrophils expressed $\mathrm{HCA}_{2}$. Purified $(\mathbf{A}-\mathbf{C})$ peritoneal neutrophils and $(\mathbf{D}-\mathbf{F})$ spinal cord sections from Hca2mRFP reporter mice subjected to $E A E$ were stained with $(B)$ anti-Ly-6G and (E) anti-PMN (Ly-6G/Ly-6C) antibodies specific for neutrophils (green). (A and $\mathbf{D}$ ) mRFP signal (red) represents $\mathrm{HCA}_{2}$ expression. Arrows indicate neutrophils stained by anti-PMN and expressing $\mathrm{HCA}_{2}$. Asterisks indicate mRFP+ and anti-PMN- cells most likely representing macrophages or microglia. Scale bar: $40 \mu \mathrm{m}$. (G) MMF $(100 \mu \mathrm{M})$ reduced the adhesion of wildtype neutrophils but not of $\mathrm{Hca}^{-/-}$neutrophils to TNF-stimulated bEnd. 3 cells. Values are mean $\pm \operatorname{SEM}(n=4)$ in 1 of 2 experiments with similar results. $P<0.05$ (2-way ANOVA for drug treatment); ${ }^{*} P<0.05$ (Bonferroni post-hoc test). (H) MMF inhibited the CXCL2-induced migration in wild-type neutrophils but not in $\mathrm{HCa}^{-/-}$neutrophils. Values are mean $\pm \operatorname{SEM}(n=3-4)$ in 1 of 3 experiments with similar results. $P=0.05$ (2-way ANOVA for drug treatment); ${ }^{*} P<0.05$ (Bonferroni post-hoc test). type cells (Supplemental Figure 4). In $\mathrm{Hca}^{-/-}$macrophages, MMF did not stimulate Nqo1 expression (Supplemental Figure 4B), but otherwise induction was not impaired in $\mathrm{Hca}^{-/-}$cells (Supplemental Figure 4, A, C, and D), demonstrating that MMF exerts $\mathrm{HCA}_{2}$-independent effects in addition to the $\mathrm{HCA}_{2}$-dependent reduction in neutrophil adhesion and chemotaxis.

In summary, we obtained evidence for the first time that the protective effect of DMF in EAE is mediated by $\mathrm{HCA}_{2}$. This finding does not exclude additional actions of DMF in EAE and MS. DMF or its metabolite MMF may directly form conjugates with the NRF2 inhibitor KEAP-1 or glutathione to induce NQO1 and HMOX1 gene expression $(6,7)$. Notably, we found that the induction of Nqo1 and Hmox 1 was largely $\mathrm{HCA}_{2}$-independent, whereas the improvement of the clinical outcome and the neutrophil recruitment did depend on $\mathrm{HCA}_{2}$. The conclusion from our study that $\mathrm{HCA}_{2}$ mediates the protective effect of DMF in EAE and MS is supported by previous data. First, DMF and another chemically unrelated agonist of $\mathrm{HCA}_{2}$, nicotinic acid, share various clinical effects, including the well-known cutaneous flushing response. In mice, there is direct evidence that $\mathrm{HCA}_{2}$ mediates the DMF-induced cutaneous flushing (16), suggesting that $\mathrm{HCA}_{2}$ is activated by DMF treatment of patients with MS. Second, it has been reported that nicotinic acid ameliorates EAE pathology (20). This effect of nicotinic acid, a prototypical agonist of $\mathrm{HCA}_{2}$, is well explained by the pivotal role of $\mathrm{HCA}_{2}$.

Concerning the potential cellular mode of action, it is interesting to note that $\mathrm{HCA}_{2}$ expression is high in human neutrophils $(11,19)$. Although the role of neutrophils in MS is not fully understood, it is clear that neutrophils show a primed phenotype in patients with MS (21). Moreover, neutrophils are involved in disease progression of EAE (12-15). $\mathrm{G}_{\mathrm{i}}$-coupled receptors are known to modulate chemotaxis of neutrophils (22). More specifically, $\mathrm{HCA}_{2}$ activates the protein kinase ERK (23) that is able to transmit a "stop" signal for neutrophil migration (22). By interfering with neutrophil recruitment, DMF may exert secondary effects on other immune cells in EAE and MS (24). Importantly, the identification of $\mathrm{HCA}_{2}$ as a drug target in EAE and MS opens up a rich therapeutic armamentarium. Several structurally diverse agonists of $\mathrm{HCA}_{2}$ are already available, which may help to further optimize therapy $(25,26)$.

\section{Methods}

Animals. Female mice aged 8 to 12 weeks were used for in vivo experiments. As $\mathrm{Hca}_{2}^{-/-}$mice (10) were backcrossed with C57BL/6 mice for at least 8 generations, we used C57BL/ 6 mice as wild-type controls, if not indicated otherwise. Hca ${ }^{\text {mRFP }}$ (Gpr109 $\mathrm{a}^{\mathrm{mRFP}}$ ) mice have been described previously (16).

Induction of EAE. Active EAE was induced by subcutaneous immunization with $\mathrm{MOG}_{35-55}$ peptide $(100 \mu \mathrm{g})$ emulsified in incomplete Freund's adjuvant (BD Difco) containing Mycobacterium tuberculosis (400 $\mu \mathrm{g}$, BD Difco). In addition, mice were treated with pertussis toxin (PTX; 200 ng, List Biological Laboratories) administered intraperitoneally on the day of immunization (day 0) and on dpi 2. In the experiments depicted in Figure 1, A-D, and Supplemental Figure 1B, we used the EAE Induction Hooke Kit (MOG ${ }_{35-55} / \mathrm{CFA}$ Emulsion PTX, Hooke Labs) according to the manufacturer's instructions. Animals were examined daily and scored as follows: 0, no disease; 0.5 , tail weakness; 1 , limp tail; 2 , hind limb weakness; 2.5 , partial hind limb paralysis; 3 , hind limb paralysis; 3.5 , hind limb paralyzed and 
weakness in forelimbs; 4, forelimb paralyzed; and 5, moribund. Paralyzed animals were given easier access to food and water. Mice were treated with vehicle or DMF (30 mg/kg body weight, twice per day, Sigma-Aldrich) that was suspended in $0.8 \%$ Methocel (Sigma-Aldrich Fluka). Starting on dpi 3, vehicle $(0.8 \%$ Methocel) or DMF was given by gavage until the end of the experiments. The therapeutic time window of DMF probably started only between dpi 5 and 7, because PTX treatment of mice on day 0 and dpi 2 reduced the inhibitory effect of MMF on neutrophil adhesion in vitro until dpi 5 (Supplemental Figure 5). This effect is explained by the fact that PTX irreversibly $\mathrm{ADP}$ ribosylates $\mathrm{G}_{\mathrm{i}}$ proteins and consequently interferes with the actions of the $\mathrm{G}_{\mathrm{i}}$-coupled $\mathrm{HCA}_{2}$ (10). In all experiments, investigators were blinded to the genotype of mice or to both genotype and treatment.

Supplemental Methods. Additional procedures and details are described in the Supplemental Methods.

Statistics. EAE scores were evaluated by using the nonparametric MannWhitney or Kruskal-Wallis tests. Histology, neutrophil migration, adhesion assays, gene expression, and neutrophil viability were analyzed by 2 -way ANOVA with Bonferroni post-hoc test, with the only exception of the concentration-response relationship (Supplemental Figure 2), which was analyzed with 1-way ANOVA followed by Bonferroni post-hoc test. As indicated in the figure legends, Student's $t$ test with Bonferroni correction was used in flow cytometry experiments. $P$ values of less than 0.05 were considered significant.
Study approval. All experiments were performed according to animal protection law in Germany and approved by the local animal welfare authorities (Regierungspräsidium Karlsruhe; Ministerium für Energiewende, Landwirtschaft, Umwelt und ländliche Räume, Kiel, Germany).

\section{Acknowledgments}

We thank Cornelia Magnussen and Ines Stölting for expert help. The research leading to these results received funding from the European Union's Seventh Framework Program FP7 under grant agreement 607962 (nEUROinflammation). N. Wettschureck was supported by the CRC 128 of the Deutsche Forschungsgemeinschaft. Furthermore, we acknowledge the support from the China Scholarship Council to H. Chen.

Received for publication July 22, 2013, and accepted in revised form January 30, 2014.

Address correspondence to: Markus Schwaninger, Institute of Experimental and Clinical Pharmacology and Toxicology, University of Lübeck, Ratzeburger Allee 160, 23538 Lübeck, Germany. Phone: 49.451.5002681; Fax: 49.451.5003327; E-mail: markus.schwaninger@pharma.uni-luebeck.de.
1. Fox RJ, et al. Placebo-controlled phase 3 study of oral BG-12 or glatiramer in multiple sclerosis. NEngl J Med. 2012;367(12):1087-1097.

2. Gold R, et al. Placebo-controlled phase 3 study of oral BG-12 for relapsing multiple sclerosis. N Engl J Med. 2012;367(12):1098-1107.

3. Reich K, Thaci D, Mrowietz U, Kamps A, Neureither M, Luger T. Efficacy and safety of fumaric acid esters in the long-term treatment of psoriasis - a retrospective study (FUTURE). J Dtsch Dermatol Ges. 2009;7(7):603-611.

4. Croxford AL, Kurschus FC, Waisman A. Mouse models for multiple sclerosis: historical facts and future implications. Biochim Biophys Acta. 2011;1812(2):177-183.

5. Schilling S, Goelz S, Linker R, Luehder F, Gold R. Fumaric acid esters are effective in chronic experimental autoimmune encephalomyelitis and suppress macrophage infiltration. Clin Exp Immunol. 2006;145(1):101-107.

6. Linker RA, et al. Fumaric acid esters exert neuroprotective effects in neuroinflammation via activation of the Nrf2 antioxidant pathway. Brain. 2011; 134(pt 3):678-692.

7. Ghoreschi K, et al. Fumarates improve psoriasis and multiple sclerosis by inducing type II dendritic cells. J Exp Med. 2011;208(11):2291-2303.

8. Tang H, Lu JY, Zheng X, Yang Y, Reagan JD. The psoriasis drug monomethylfumarate is a potent nicotinic acid receptor agonist. Biochem Biophys Res Commun. 2008;375(4):562-565.

9. Lukasova M, Hanson J, Tunaru S, Offermanns S. Nicotinic acid (niacin): new lipid-independent mechanisms of action and therapeutic potentials.
Trends Pharmacol Sci. 2011;32(12):700-707.

10. Tunaru S, et al. PUMA-G and HM74 are receptors for nicotinic acid and mediate its anti-lipolytic effect. Nat Med. 2003;9(3):352-355.

11. Maciejewski-Lenoir D, Richman JG, Hakak Y, Gaidarov I, Behan DP, Connolly DT. Langerhans cells release prostaglandin D2 in response to nicotinic acid. J Invest Dermatol. 2006;126(12):2637-2646.

12. Carlson T, Kroenke M, Rao P, Lane TE, Segal B. The Th17-ELR+ CXC chemokine pathway is essential for the development of central nervous system autoimmune disease. J Exp Med. 2008;205(4):811-823.

13. Liu L, Darnall L, Hu T, Choi K, Lane TE, Ransohoff RM. Myelin repair is accelerated by inactivating CXCR2 on nonhematopoietic cells. J Neurosci. 2010 30(27):9074-9083.

14. McColl SR, Staykova MA, Wozniak A, Fordham $\mathrm{S}$, Bruce J, Willenborg DO. Treatment with antigranulocyte antibodies inhibits the effector phase of experimental autoimmune encephalomyelitis. Jimmunol. 1998;161(11):6421-6426.

15. Maatta JA, Sjoholm UR, Nygardas PT, Salmi AA, Hinkkanen AE. Neutrophils secreting tumor necrosis factor alpha infiltrate the central nervous system of BALB/c mice with experimental autoimmune encephalomyelitis. J Neuroimmunol. 1998; 90(2):162-175

16. Hanson J, et al. Nicotinic acid- and monomethyl fumarate-induced flushing involves GPR109A expressed by keratinocytes and COX-2-dependent prostanoid formation in mice. J Clin Invest. 2010; 120(8):2910-2919.

17. Murikinati $S$, et al. Activation of cannabinoid 2 receptors protects against cerebral ischemia by inhibiting neutrophil recruitment. FASEB J. 2010; 24(3):788-798

18. Kees F. Dimethyl fumarate: a Janus-faced substance? Expert Opin Pharmacother. 2013; 14(11):1559-1567.

19. Kostylina G, Simon D, Fey MF, Yousefi S, Simon HU. Neutrophil apoptosis mediated by nicotinic acid receptors (GPR109A). Cell Death Differ. 2008; 15(1):134-142.

20. Zhang J, et al. Niaspan treatment improves neurological functional recovery in experimental autoimmune encephalomyelitis mice. Neurobiol Dis. 2008; 32(2):273-280

21. Naegele M, Tillack K, Reinhardt S, Schippling S, Martin R, Sospedra M. Neutrophils in multiple sclerosis are characterized by a primed phenotype. J Neuroimmunol. 2012;242(1-2):60-71.

22. Kolaczkowska E, Kubes P. Neutrophil recruitment and function in health and inflammation. Nat Rev Immunol. 2013;13(3):159-175.

23. Walters RW, et al. $\beta$-Arrestin 1 mediates nicotinic acid-induced flushing, but not its antilipolytic effect, in mice. J Clin Invest. 2009;119(5):1312-1321.

24. Ransohoff RM, Brown MA. Innate immunity in the central nervous system. J Clin Invest. 2012; 122(4):1164-1171.

25. Shen HC, Colletti SL. High-affinity niacin receptor GPR109A agonists. Annu Rep Med Chem. 2010;45:72-94.

26. Offermanns $S$, et al. International Union of Basic and Clinical Pharmacology. LXXXII: Nomenclature and Classification of Hydroxy-carboxylic Acid Receptors (GPR81, GPR109A, and GPR109B). Pharmacol Rev. 2011;63(2):269-290. 